\title{
Some exact values for the diameter in Cayley graph $H_{\ell, p}$
}

\author{
Caroline Patrão $^{123}$, Luis Kowada ${ }^{4}$, Diane Castonguay ${ }^{5}$, André Ribeiro $^{6}$, Celina de Figueiredo ${ }^{1}$ \\ ${ }^{1}$ Programa de Engenharia de Sistemas e Computação - COPPE \\ Universidade Federal do Rio de Janeiro (UFRJ) \\ Caixa Postal 68.511 - 21.941-972 - Rio de Janeiro - RJ - Brazil \\ ${ }^{2}$ Instituto Federal do Rio de Janeiro (IFRJ) - Nilópolis, RJ - Brazil \\ ${ }^{3}$ Instituto Federal de Goiás (IFG) - Formosa, GO - Brazil \\ ${ }^{4}$ Instituto de Computação \\ Universidade Federal Fluminense (UFF) - Niterói, RJ - Brazil \\ ${ }^{5}$ Instituto de Informática \\ Universidade Federal de Goiás (UFG) - Goiânia, GO - Brazil \\ ${ }^{6}$ Instituto Federal Goiano (IF Goiano) - Rio Verde, GO - Brazil \\ caroline.patraodifrj.edu.br, luisdic.uff.br, dianedinf.ufg.br \\ andre. cunha@ifgoiano.edu.br celina@cos.ufrj.br
}

\begin{abstract}
The family of graphs $H_{\ell, p}$ has been defined in the context of edge partitions. The established properties such as vertex transitivity and low diameter suggest this family as a good topology for the design of interconnection networks. The vertices of the graph $H_{\ell, p}$ are the $\ell$-tuples with values between 0 and $p-1$, such that the sum of the $\ell$ values is a multiple of $p$, and there is an edge between two vertices, if the two corresponding tuples have two pairs of entries whose values differ by one unit. In order to work towards the diameter, the difference between an upper and a lower bounds is established to be at most $\ell+p$ and we present subfamilies of graphs $H_{\ell, p}$ such that, for several values of $\ell$ and $p$, the bounds are tight.
\end{abstract}

\section{Introduction}

In this work, we are motivated by the design and analysis of static networks. Static networks can be modeled using tools from Graph Theory. A graph represents an interconnection network, where the processors are the vertices and the communication links between processors are the edges connecting the vertices. There are several parameters of interest to specify a network: for instance low degree and low diameter. The degree relates to the port capacity of the processors and hence to the hardware cost of the network. The maximum communication delay between a pair of processors in a network is measured by the diameter of the graph. Thus, the diameter is a measure of the running cost. Cayley graphs are connected vertex-transitive graphs that provide two challenging and extensively studied problems: Hamiltonian cycle and graph diameter. Cayley graphs are regular, in some cases have logarithmic diameter, and can be used to design interconnection networks [Vadapalli and Srimani 1996].

This paper is organized as follows: In Section 2, the definition of Cayley graphs is introduced to explain the concept of abstract groups, which are described by a generating 
set and we define the graph $H_{\ell, p}$. Section 3 is devoted to describe exact formulas for the diameter of subfamilies of graphs $H_{\ell, p}$ such that the known bounds are reached as well as the values between them.

\section{The Cayley Graph $H_{\ell, p}$}

Let $(G,+)$ be a group and $C \subseteq G$ be a generating set of $G$ in which any element of $G$ can be obtained from elements of $C$ by a finite number of applications of the operation + . A directed graph $\Gamma=(V, E)$ is a Cayley graph for a group $(G,+)$ with a generating set $C$, if there is a bijection mapping each $x \in V$ to an element $g_{x} \in G$, such that $x y$ is a directed edge of $E$ if and only if there exists $c \in C$ such that $g_{y}=c+g_{x}$. If the identity element $\iota \notin C$, then there are no loops in $\Gamma$, and $\Gamma$ satisfies the identity free property. If $g \in G$ then there is a unique $g^{\prime} \in G$ such that $g+g^{\prime}=\iota$, denote $g^{\prime}$ by $-g$ and in this case we define $g_{x}-g_{y}=g_{x}+\left(-g_{y}\right)$. If $c \in C$ implies $-c \in C$, then for every edge from $g$ to $g+c$, there is also an edge from $g+c$ to $(g+c)+(-c)=g$, and $\Gamma$ satisfies the symmetry condition. A Cayley graph that satisfies both the identity free property and the symmetry condition is an undirected graph. The graphs $H_{\ell, p}$ considered in this paper are undirected Cayley graphs associated to the group $\left(V_{\ell, p},+\right)$ with generating set $C_{\ell, p}$ [Ribeiro et al. 2010].

For each $\ell \geq 3$ and $p \geq 3$, Holyer [Holyer 1981] defines a graph $H_{\ell, p}=$ $\left(V_{\ell, p}, E_{\ell, p}\right)$ where

$$
V_{\ell, p}=\left\{x=\left(x_{1}, \ldots, x_{\ell}\right), \text { with } x_{k} \in \mathbb{Z}_{p} \text { and } \sum_{k=1}^{\ell} x_{k} \equiv_{p} 0\right\},
$$

$E_{\ell, p}=\left\{x y:\right.$ there are distinct $i, j$ such that $y_{k} \equiv_{p} x_{k}$ for $k \neq i, j$ and $y_{i} \equiv_{p}$ $\left.x_{i}+1, \quad y_{j} \equiv_{p} x_{j}-1\right\}$.

The vertices of $H_{\ell, p}$ are the elements of a finite group $\left(V_{\ell, p},+\right)$, where the operation + is such that $x+y=\left(x_{1}, \ldots, x_{\ell}\right)+\left(y_{1}, \ldots, y_{\ell}\right)=\left(x_{1}+y_{1}, \ldots, x_{\ell}+y_{\ell}\right)$, where $x_{k}+y_{k}$ is the operation in $\left(\mathbb{Z}_{p},+\right)$, for $x, y \in V_{\ell, p}$ and the element $(0,0, \ldots, 0,0) \in V_{\ell, p}$ is the identity vertex $\iota$. The generating set $C_{\ell, p}$ is the set of $\ell$-tuples $e_{i, j}=\left(c_{1}, \ldots, c_{\ell}\right)$ such that, for $i, j \in\{1, \ldots \ell\}$ with $i \neq j$, we have that $c_{k}=1$ when $k=i, c_{k}=p-1$ when $k=j$, and otherwise $c_{k}=0$.

\section{Diameter of Graph $\boldsymbol{H}_{\ell, p}$}

As the graph $H_{\ell, p}$ has $p^{\ell-1}$ vertices, Castonguay et al.[Castonguay et al. 2015] highlighted some special vertices to study the diameter of the graph $H_{\ell, p}$ and obtained an upper bound for this diameter to be $\left\lfloor\frac{\ell \cdot p}{4}\right\rfloor$. The diameter can be found by the greatest value of the distance from each special vertex to the identity vertex $\iota$. For the sake of completeness, we rewrite the results presented in [Castonguay et al. 2015, Lemma 11] as $D\left(H_{\ell, p}\right)=$ $\max _{t}\left\{(\ell-t) \cdot\left\lfloor\frac{t \cdot p}{\ell}\right\rfloor\right\}$, for $0 \leq t<\ell$.

Note that a lower bound for the diameter of $H_{\ell, p}$ is found when $t=\left\lfloor\frac{\ell}{2}\right\rfloor$, thus the solution of the diameter problem for the graph $H_{\ell, p}$ is $\Theta(\ell \cdot p)$.

Proposition 1 presents the difference between the known bounds on the diameter of the graph $H_{\ell, p}$. 
Proposition 1. The difference between an upper and a lower bounds $\left\lfloor\frac{\ell \cdot p}{4}\right\rfloor-$ $\left(\ell-\left\lfloor\frac{\ell}{2}\right\rfloor\right)\left\lfloor\frac{\left\lfloor\frac{\ell}{2}\right\rfloor \cdot p}{\ell}\right\rfloor$ is at most $\ell+p$.

Proof. We omit in the present extended abstract the explicit calculations.

Table 1 presents the specific cases for which these bounds are known to be tight. [Castonguay et al. 2015].

Table 1. Exact known values on the diameter

\begin{tabular}{|c|c|c|c|}
\hline Graph $H_{\ell, p}$ & Lower Bound Reached & Upper Bound Reached & Diameter \\
\hline$\ell$ and $p$ even & always & always & $\frac{\ell \cdot p}{4}$ \\
\hline$p$ is a multiple of $\ell$ & always & when $\ell$ is even & $\frac{p \cdot\left\lfloor\frac{\ell}{2}\right\rfloor \cdot\left\lceil\frac{\ell}{2}\right\rceil}{\ell}$ \\
\hline$p$ is a multiple of $\ell$ odd & always & never & $\frac{p \cdot \ell}{4}-\frac{p}{4 \cdot \ell}$ \\
\hline$p=\ell$ and $p=\ell+1$ & always & when $p=\ell$ is even & $\left\lfloor\frac{\ell}{2}\right\rfloor \cdot\left\lceil\frac{\ell}{2}\right\rceil$ \\
\hline$\ell=2$ & always & when $p$ is even & $\left\lfloor\frac{p}{2}\right\rfloor$ \\
\hline$\ell=3$ & always & never & $\left\lfloor\frac{2 \cdot p}{3}\right\rfloor$ \\
\hline$\ell=4$ & always & when $p$ is even & $2 \cdot\left\lfloor\frac{p}{2}\right\rfloor$ \\
\hline
\end{tabular}

Propositions 2 and 3 present two subfamilies of graphs $H_{\ell, p}$ for which we are able to establish new exact values on the diameter.

Proposition 2. Let $z, h \in \mathbb{Z}_{+}$, so $D\left(H_{2 \cdot z, h \cdot z}\right)= \begin{cases}\left\lfloor\frac{z^{2}}{2}\right\rfloor \cdot h, & \text { if } z \geq h \text { and } h \text { is odd; } \\ \left\lfloor\frac{h z}{2}\right\rfloor \cdot z, & \text { otherwise. }\end{cases}$

Proof. Since $\ell=2 z$ and $p=h z$, we have that $D\left(H_{2 \cdot z, h \cdot z}\right)=\max \left\{(2 z-t)\left\lfloor\frac{t \cdot h}{2}\right\rfloor\right\}$, for $0 \leq t<\ell$. Considering $h$ or $z$ even, we have that $\ell$ and $p$ are even, by Table 1 , $D\left(H_{2 z, h z}\right)=\frac{2 z \cdot h z}{4}=\frac{h z^{2}}{2}$. Clearly, if $h$ and $z$ are even, then $D\left(H_{2 z, h z}\right)=\frac{h z^{2}}{2}$. As we desired, $\left\lfloor\frac{h z}{2}\right\rfloor \cdot z=\frac{h z^{2}}{2}$. We omit the proof for $h$ and $z$ odd considering the parity of $t$ in this extended abstract, however we find that $D\left(H_{2 z, h z}\right)=\max \left\{\frac{h \cdot z^{2}-z}{2}, \frac{h \cdot z^{2}-h}{2}\right\}$. Therefore, if $z \geq h$ then $D\left(H_{2 z, h z}\right)=\frac{h \cdot z^{2}-h}{2}=\frac{h \cdot\left(z^{2}-1\right)}{2}=\left\lfloor\frac{z^{2}}{2}\right\rfloor \cdot h$. If $z<h$ then $D\left(H_{2 z, h z}\right)=\frac{h \cdot z^{2}-z}{2}=\frac{z \cdot(h z-1)}{2}=\left\lfloor\frac{h z}{2}\right\rfloor \cdot z$.

Proposition 3. Let $z, h \in \mathbb{Z}_{+}$, we have that

$$
D\left(H_{4 \cdot z, h \cdot z}\right)= \begin{cases}z^{2} \cdot h-z, & \text { if } z<\frac{h-1}{2} \text { and } h, z \text { are odd } \\ \frac{4 z^{2} \cdot h-2 z-h+1}{4}, & \text { if } \frac{h-1}{2} \leq z \leq \frac{3 h+1}{2} \text { and } h, z \text { are odd } \\ z^{2} \cdot h-h, & \text { if } z>\frac{3 h+1}{2} \text { and } h, z \text { are odd } \\ z^{2} \cdot h, & \text { otherwise. }\end{cases}
$$


Proof. Since $\ell=4 z$ and $p=h z$, we have that $D\left(H_{4 z, h z}\right)=\max \left\{(4 z-t)\left\lfloor\frac{t h}{4}\right\rfloor\right\}$ for $0 \leq$ $t<\ell$. Considering $h$ or $z$ even, we have that $\ell$ and $p$ are even, by Table $1, D\left(H_{4 z, h z}\right)=$ $\frac{4 z \cdot h z}{4}=h \cdot z^{2}$.

Considering $h$ and $z$ odd, we have five cases. We omit the proof for $t h, t h-$ $1, t h-2, t h-3$ and $t h-4$ multiple of 4 in this extended abstract, however we find that $D\left(H_{4 z, h z}\right)=\max \left\{\frac{4 z^{2} \cdot h-2 z-h+1}{4}, \frac{4 z^{2} \cdot h-6 z-h+3}{4}, z^{2} \cdot h-h, z^{2} \cdot h-z\right\}=z^{2} \cdot h+$ $\min \left\{\frac{2 z+h-1}{4}, \frac{6 z+h-3}{4}, h, z\right\}$. Comparing these results, we have that if $\frac{6 z+h-3}{4}<\frac{2 z+h-1}{4}$ then $z<\frac{1}{2}$. So, the expression $\frac{6 z+h-3}{4}$ can be regarded. Note that if $\frac{2 z+h-1}{4} \leq h$ then $z \leq$ $\frac{3 h+1}{2}$, and if $\frac{2 z+h-1}{4} \leq z$ then $z \geq \frac{h-1}{2}$. So, for $z$ and $h$ odd: $D\left(H_{4 z, h z}\right)=\frac{4 z^{2} \cdot h-2 z-h+1}{4}$ if $\frac{h-1}{2} \leq z \leq \frac{3 h+1}{2}$, and $D\left(H_{4 z, h z}\right)=z^{2} \cdot h-z$, if $z>\frac{h-1}{2}$ and $D\left(H_{4 z, h z}\right)=z^{2} \cdot h-h$, if $z>\frac{3 h+1}{2}$. This concludes the proof.

In Table 2 we can see how these new results can be found based on the obtained bounds as well as the values between them.

Table 2. New exact values on the diameter.

\begin{tabular}{|c|c|c|c|c|}
\hline Graph $H_{\ell, p}$ & Lower Bound & Upper Bound & Diameter & $\begin{array}{c}\text { Neither lower } \\
\text { nor upper bounds }\end{array}$ \\
\hline $\begin{array}{c}\ell=2 \cdot z, p=h \cdot z \\
\text { where } z, h \in \mathbb{Z}_{+}\end{array}$ & $\begin{array}{c}\text { If } z \leq h \text { and } h, z \text { odd; } \\
\text { or if } h \text { even; or if } z \text { even }\end{array}$ & $\begin{array}{c}\text { If } z \text { even; } \\
\text { or if } h=1 ; \\
\text { or } h \text { even }\end{array}$ & $\begin{array}{c}\text { It depends on } \\
z \text { and } h\end{array}$ & If $z>h$ and $h, z$ odd \\
\hline $\begin{array}{c}\ell=4 \cdot z, p=h \cdot z \\
\text { where } z, h \in \mathbb{Z}_{+}\end{array}$ & $\begin{array}{c}\text { If } z \leq \frac{h-1}{2} \text { and } h, z \text { odd; } \\
\text { or if } h \text { even; or if } z \text { even }\end{array}$ & $\begin{array}{c}\text { If } z \text { even; } \\
\text { or } h \text { even }\end{array}$ & $\begin{array}{c}\text { It depends on } \\
z \text { and } h\end{array}$ & $\begin{array}{c}\text { If } z>\frac{3 h+1}{2} \text { and } h, z \text { odd; } \\
\text { or if } \frac{h-1}{2}<z<\frac{3 h+1}{2}\end{array}$ \\
\hline
\end{tabular}

\section{Conclusion}

Several authors observed that Cayley graphs provide a useful and unified framework for the design of interconnection networks for parallel computers. For the family of graphs $H_{\ell, p}$, we obtain new results for the diameter of subfamilies of graphs $H_{\ell, p}$ that show that the known bounds for the diameter are tight. This established property makes the Cayley graph $H_{\ell, p}$ a good scheme of interconnection networks.

\section{References}

Castonguay, D., Ribeiro, A. C., Figueiredo, C. M. H., and Kowada, L. A. B. (2015). On the diameter of the Cayley Graph $H_{\ell, p}$. Matemática Contemporânea, 44:1-10.

Holyer, I. (1981). The NP-Completeness of Some Edge-Partition Problems. SIAM Journal Computing, 10(4):713-717.

Ribeiro, A. C., Figueiredo, C. M. H., and Kowada, L. A. B. (2010). An evidence for Lovász conjecture about Hamiltonian paths and cycles. Matemática Contemporânea, 39:121-128.

Vadapalli, P. and Srimani, P. K. (1996). A New Family of Cayley Graph Interconnection Networks of Constant Degree Four. IEEE Transactions on Parallel and Distributed Systems, 7(1):26-32. 